\title{
Transversus abdominis plan bloğu için ultrason eşliğinde farklı bölgelerden yapılan ölçümler
}

Ultrasound measurements from different regions for transversus abdominis plan block

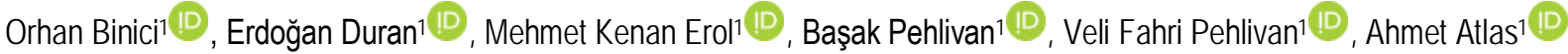 \\ ${ }^{1}$ Anesteziyoloji ve Reanimasyon AB, Harran Üniversitesi Tıp Fakültesi, Şanlıurfa, Türkiye
}

\section{Öz.}

Amaç: Analjeziklerin yetersiz veya yüksek doz kullanımı giderek önem kazanmaktadır; bu yüzden analjezi yöntemleri her gün geçtikçe gelişmektedir. Transversus abdominis plan (TAP) bloğu ultrason eşliğinde batın cerrahilerinde ağıı kontrolü için yapılan bir yöntemdir. Çalışmamızda Ultrason eşliğinde farklı bölgelerden ölçümler alarak TAP bloğunun hangi bölgeden uygulanması gerektiğini araştırmayı amaçladık.

Materyal ve Metot: Çalışmaya 18-65 yaş arası 30 hasta çalışmaya alındı. Ön karın bölgesinden ultrasonun lineer probu subkostal bölgeden ve krista iliyakanın üstünden anterior aksiler bölge ve mediyal aksiler hatta denk gelecek bölgeden internal oblık kas, eksternal oblik kas ve transversus abdominal plan kllıf genişliği kaydedildi. Yine bu bölgelerin görüntü netliği (kötü, iyi ve çok iyi olarak ) değerlendirilip kaydedildi.

Bulgular: Krista iliyaka üzerinden anterior aksiler hattın (A1) median aksiler hat (A2) ile karşılaştırımasında alınan ölçümlerde internal oblik kas, eksternal oblik kas, transversus abdominus kası genişlikleri ve transversus abdominus fasiya daha düşük çıkmış ve istatistiksel olarak olarak anlamlı bulunmuştur $(P<0,05)$. Kostal kenar altından anterior aksiler hattın (B1) median aksiler hat (B2) ile karşılaştırılmasında alınan ölçümlerde internal oblık kas ile transversus abdominus fasiya daha düşük çıkmış ve istatistiksel olarak anlamlı bulunmuştur $(P<0,05)$.

Sonuç: Medial aksiler hatta anterior aksiler hatta göre ölçümlerin daha yüksek çıkması ve görüntü netliğinin daha iyi olması nedeniyle TAP blok uygulanırken probun mediyal aksiler hat üzerinden yapılması gerektiği kanaatindeyiz.

Anahtar Kelimeler: Transversus abdominis plan bloğu, Ultrason, Analjezi.

\section{Abstract}

Background: Inadequate or overdose use of analgesics is becoming increasingly important; therefore, analgesia methods are developing day by day. Transversus abdominis plan (TAP) block is a method for pain control in abdominal surgeries under ultrasound guidance. In our study, we aimed to investigate the area where TAP block should be applied by taking measurements from different regions under ultrasound guidance.

Methods: Thirty patients aged between 18-65 years were included in the study. Linear oblique muscle, external oblique muscle, and transverse abdominal plan sheath width from the anterior axillary region and medial axillary line from the anterior abdominal region, from the subcostal region of the ultrasound to the anterior abdomen, and from the upper iliac crest were recorded. The image sharpness of these regions (poor, good and very good) was evaluated and recorded.

Results: Internal oblique muscle, external oblique muscle, transversus abdominus muscle widths and transversus abdominus fascia were found to be lower and statistically significant $(P<0)$ when comparing the anterior axillary line (A1) over Krista iliac line with the median axillary line (A2). , 05). Internal oblique muscle and transversus abdominus fascia were found to be lower in the measurements taken when comparing the anterior axillary line (B1) under the costal edge with the median axillary line (B2) and were found to be statistically significant $(\mathrm{P}<0.05)$.

Conclusion: We believe that the probe should be made over the medial axillary line when TAP block is applied because the measurements are higher than the anterior axillary line and the image clarity is better.

Key words: Transversus abdominis plan block, Ultrasound, Analgesia.

\section{Sorumlu Yazar I \\ Corresponding Author}

Dr. Orhan Binici

Anesteziyoloji ve Reanimasyon $A B$ Harran Üniversitesi Tıp Fakültesi, Şanlıurfa, Türkiye

Tel: +90 5052224717

E-mail: orhan_binici@windowslive.com Geliş tarihi / Received: 01.06.2019 Kabul tarihi / Accepted: 23.07.2019

DOI: $10.354440 /$ hutfd. 573334 


\section{Giriş}

Postoperatif ve intraoperatif analjezi kontrolü hastaların iyileşme ve tedaviye vereceği yanıt ve bakımından önem arz etmektedir. Transversus abdominis plan (TAP) bloğu intraoperatif ve postoperatif analjezi sağlamak için karnın antero-lateral bölgesinden lokal anestezik ajan uygulanılarak yapılan rejyonel anestezi tekniklerinden biridir. Rafi tarafından ilk kez tanımlanan bu blok 2007 ylında Hebbart ve ark. tarafından Ultrason (Usg) eşliğinde uygulanmış; ve şu an günümüzde batın cerrahilerinde Usg eşliğinde analjezi amacıyla sık kullanılan bir yöntem olmuştur. Bu yöntemle internal oblik ve transversus abdominis kas arasındaki fasiyal boşluğa lokal anestezik verilerek torakal inter-kostal (T7-T12) ve birinci lomber (L1) sinirlerin ön dallarını bloke edilir (1-3). Bu konu çok araştırımakla beraber iğne giriş yeri, verilecek lokal anesteziğin volümü, verildiği bölgede yayılım detayları hala araştırımakta ve bu konuda çalışmalar devam etmektedir. Bu blok uygulanılan cerrahinin yerine göre kostal kenarın altı ve krista iliyakanın üstü arasından ve aksiler hatta denk gelecek bölgeden yapılması tavsiye edilmektedir $(4,5)$. Çalışmamızda Usg eşliğinde, tanımlanan bölge arasından anterior ve orta aksiler hattan batın kaslarının ölçümlerini alarak birbirleri ile karşılaştırmayı amaçladık.

\section{Materyal ve Metot}

Etik kurul onayı ve hastaların onamı alındıktan sonra ASA I-II (American Society of Anesthesiologists) grubuna giren 18-65 yaş arası 30 hasta çalışmaya alındı. Karın ön bölgesinden cerrahi operasyon geçiren, ultrason probunun bırakıldığı yerde enfeksiyon ve travması olan ve BMl (body mass index) $>30$ hastalar çalışma dışı bırakıldı. Kişilerin yaşları, cinsiyeti, BMI kaydedildi.

Ön karın bölgesinden ultrason'nun (Esaote MyLab 30 Gold, lineerprob, 10-18 MHz, Florance, Italy ) lineer probu in-plane olarak basınç uygulanmadan karın üzerine bırakılarak subkostal bölgeden anterior aksiler bölge (Şekil 1) ve mediyal aksiler hatta denk gelecek bölgeden (Şekil 2) internal oblık kas, eksternal oblik kas, ve transversus abdominal plan kılıf genişliği kaydedildi. Yine aynı şekilde spina iliyaka üzerinden anterior aksiler bölge(Şekil 3) ve orta aksiler hatta (Şekil 4) denk gelecek bölgeden internal oblik kas, eksternal oblik kas, transversus abdominus kası genişlikleri ve transversus abdominus plan kllff genişliği (Şekil 5) kaydedildi. Ölçüm alınan bölgelerden görüntünün netliği (kötü:0, iyi:1, çok iyi:3) değerlendirilerek kaydedildi. Ölçümler aynı kişi tarafından yapıldı.

\section{Bulgular}

Çalışmaya yaşları 18 ile 65 arasında değişen 30 gönüllünün subkostal ve krista iliyaka üstünden anterior aksiler bölge ve median aksiler hatta denk gelecek bölgeden karın kaslarının ve transversus abdominus fasiya'nın ölçümleri yapılmıştır. Hastaların demografik verileri Tablo 1 de verilmiştir.
Krista iliyaka üzerinden anterior aksiler hattın (A1) median aksiler hat (A2) ile karşılaştııımasında alınan ölçümlerde (Tablo 2) internal oblik kas, eksternal oblik kas, transversus abdominus kası genişlikleri ve transversus abdominus fasiya daha düşük çıkmış ve istatistiksel olarak olarak anlamlı bulunmuştur $(P<0,05)$. Kostal kenar altından anterior aksiler hattın (B1) median aksiler hat (B2) ile karşılaştırımasında alınan ölçümlerde (Tablo 2) internal oblik kas ile transversus abdominus fasiya daha düşük çıkmış ve istatistiksel olarak anlamlı bulunmuştur $(P<0,05)$.

Yine krista iliyaka üzerinden alınan ölçümlerde A2'nin (median aksiler hat) görüntüsü A1'e (aksiler hat ) göre daha iyi çıkmış ve buda istatistiksel olarak anlamlı bulunmuştur $(P<0,00)$.

Kostal kenar altından alınan ölçümlerde B2'nin (median aksiler hat) görüntüsü B1'e (aksiler hat ) göre daha iyi çıkmış ve buda istatistiksel olarak anlamlı bulunmuştur $(P<0,00)$. (Tablo 2)

Tablo 1. Demografik veriler

\begin{tabular}{ccc} 
& Frequency & Percent \\
\hline Bayan & 6 & 20.0 \\
Erkek & 24 & 80.0 \\
Total & 30 & 100.0 \\
\hline
\end{tabular}

$\underline{\text { Tablo 2. Krista iliyaka ve kostal kenar üzerinden alınan ölçümler }}$

\begin{tabular}{llllll}
\multicolumn{2}{c}{$\mathrm{n}($ (30) } & Mean & $\mathrm{N}$ & $\begin{array}{c}\text { Std. } \\
\text { Deviation }\end{array}$ & $\mathrm{P}$ \\
\hline Pair 1 & A1internal & .7100 & 30 & .11771 & .002 \\
& A2internal & .7783 & 30 & .12382 & \\
Pair 2 & A1external & 1.0197 & 30 & .17401 & .001 \\
& A2external & 1.0860 & 30 & .18286 & \\
Pair 3 & A1TAB & .4423 & 30 & .09895 & 000 \\
& A2TAB & .4803 & 30 & .10040 & \\
Pair 4 & A1Fasiya & .1363 & 30 & .03124 & 000 \\
& A2Fasiya & .1603 & 30 & .03222 & \\
Pair 5 & B1internal & .6560 & 30 & .14366 & 000 \\
& B2internal & .7397 & 30 & .15778 & \\
Pair 6 & B1external & .9631 & 30 & .17179 & .313 \\
& B2external & .9837 & 30 & .18275 & \\
Pair 7 & B1TAB & .4553 & 30 & .10718 & .304 \\
& B2TAB & .4697 & 30 & .08845 & \\
Pair 8 8 & B1Fasiya & .1250 & 30 & .03432 & .000 \\
& B2Fasiya & .1537 & 30 & .03528 & \\
Pair 9 & A1görüntü & 1.3000 & 30 & .53498 & .000 \\
& A2görüntü & 2.0000 & 30 & .00000 & \\
Pair 10 & B1görüntü & 1.2333 & 30 & .56832 & .000 \\
\hline
\end{tabular}

\section{Tartışma}

TAP blok daha önce tanımlanmasına rağmen Usg eşliğinde 2007'de ilk defa Hebbart ve ark. (3) tarafından tanımlanmışıı. Karnın anterolateral bölgesinde ciltten peritona doğru eksternal oblik kas, internal oblik kas ve transversus abdominis kasları sıralanır. Anterior abdominal duvar cilt, kaslar ve paryetal bölge T7'den T12'e kadar (altı torakal sinir) ve $L 1$ 'in ön dalları tarafından inerve edilir. Bu sinirle- 
rin terminal dalları internal oblik kas ve transversus abdominis kas arasındaki " transversus abdominis plan" adı verilen alanda karın lateral duvarı boyunca ilerler. TAP blok uygulanışı ile ilgili çalışmalar hala devam etmektedir. Hasta supin pozisyonda Usg'nin lineer probu kostal kenar ve krista iliyaka'nın arasına konarak axiller hattın denk geldiği bölgede uygun görüntü elde edildiğin de blok yapılır (69). Bizde çalışmamızda Usg eşliğinde probu kostal kenar ve krista iliyaka'nın arasına yerleştirerek anterior ve mediyal axiller hatlarda eksternal oblik kas, internal oblik kas ve transversus abdominis kasları ve transversus abdominis plan kılıfı için ölçümler aldık.

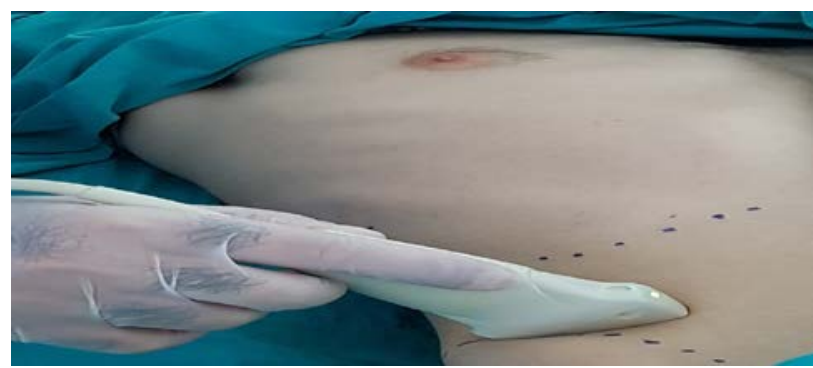

Şekil 1. Krista iliyaka üzerinden anterior aksiller hatla kesişim yeri.

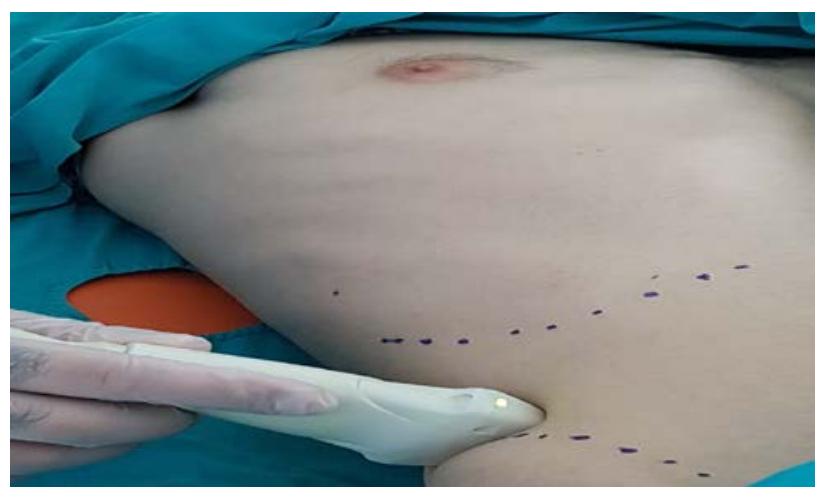

Şekil 2. Krista iliyaka üzerinden mediyal aksiller hatla kesişim yeri.

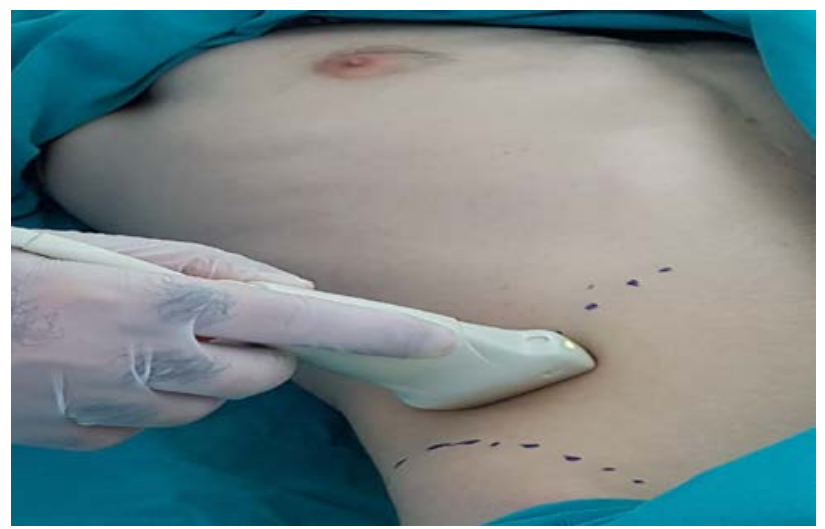

Şekil 3. Kostal kenar altından anterior aksiller hatla kesişim yeri.

Çalışmamızda krista iliyaka bölgesinde yapılan ölçümlerde; mediyal aksiler hat, anterior aksiler hatta göre eksternal oblik kas, internal oblik kas, transversus abdominis kas ve transversus abdominis kllıf genişliği daha yüksekti ve bu genişlik istatistiksel olarak anlamlıydı. Genişliğin yüksek olması bu bölgeden blok uygulamanın ve Usg eşliğinde görünürlüğünün daha belirgin ve daha kolay olduğunu göstermektedir.

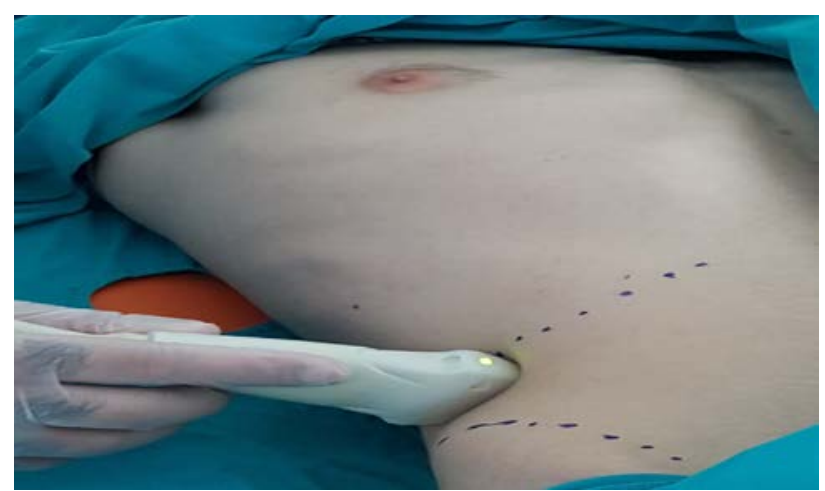

Şekil 4. Kostal kenar altından mediyal aksiller hatla kesişim yeri.

Çalışmamızda kostal kenar altından yapılan ölçümlerde; mediyal aksiler hat, anterior aksiler hatta göre internal oblik kas ve transversus abdominis kılıf genişliği daha yüksekti ve bu genişlik istatistiksel olarak anlamlıydı $(p<0,00)$. TAP blok transversus abdominis kllıf bölgesinden uygulandığından ve blok iğnesi bu bölgeden ilerletildiğinden bu bölgenin geniş olması blok uygulamasını kolaylaştırmaktadır.

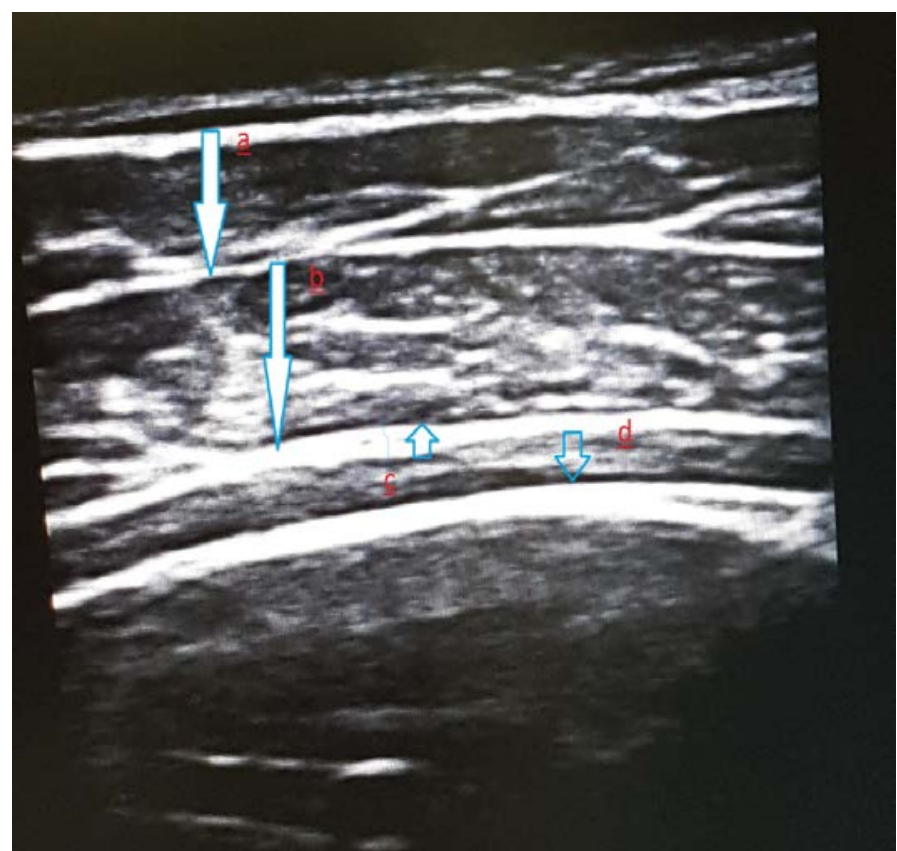

Şekil 5. a: eksternal oblik kas, b: internal oblık kas, c: transversus abdominal plan klıf genişliği, d: transversus abdominus kası.

Çalışmamızda görüntü netliğinin krista iliyaka bölgesinde ve kostal kenar altından değerlendirildiğinde; görüntü netliği medial aksiler hatta anterior aksiler hatta göre daha iyiydi ve bu istatistiksel olarak anlamlıydı $(p<0,00)$. 


\section{Sonuç}

TAP bloğun uygulanışı, iğnenin giriş bölgesi ve görüntü kalitesi ile ilgili çalışmalar hala yapılmaktadır ve bu konu çaIışmalar neticesinde netlik kazanacaktır. Usg eşliğinde ölçümler neticesinde medial aksiler hatta anterior aksiler hatta göre ölçümlerin daha yüksek çıkması ve görüntü netliğinin daha iyi olması nedeniyle TAP blok uygulanırken probun mediyal aksiler hattın üzerine bırakılarak yapılmasının daha avantajlı olacağı kanaatindeyiz.

\section{Kaynakça}

1. Ekmekçi $P$, Bengisu ZK, Kazbek BK, Has S, Tüzüner F. Ultrasound guided TAP block for the treatment of postoperative prolonged pain - an alternative approach AĞRI 2012;24(4):191-193 doi: 10.5505/agri.2012.82905

2. McDonnell J G, O'Donnell B, Curley G, Heffernan A, Laffey JG. The analgesic efficacy of transversus abdominis plane block after abdominal surgery: a prospective randomized controlled trial. Anesth Analg 2007;104(1):193-7.

3. Hebbard P, Fujiwara Y, Shibata Y, Royse C. Ultra-sound-guided transversus abdominis plane (TAP) block. Anaesth Intensive Care. 2007; 35: 616-7.

4. Gürkan Y, Tekin M. Ultrasonografi Rehberliğinde Rejyonal Anestezi. Transversus Abdominis Plan Bloğu. 2011; 145-51.

5. Theodoraki K, Papacharalampous $P$, Tsaroucha A, Vezakis A, Argyra $E$.The effect of transversus abdominis plane block on acute and chronic pain after inguinal hernia repair. A randomized controlled trial. Int J Surg. 2019 Mar;63:63-70. doi: 10.1016/j.ijsu.2019.02.007. Epub 2019 Feb 12.

6. Tekelioğlu ÜY, Demirhan A, Koçoğlu H. Transversus Abdominis Plan (TAP) Bloğu. Abant Med J 2013;2(2):156-160 doi: 10.5505/abantmedj.2013.66376

7. Mittal T, Dey A, Siddhartha R, Nali A, Sharma B, Malik V. Efficacy of ultrasound-guided transversus abdominis plane (TAP) block for postoperative analgesia in laparoscopic gastric sleeve resection: a randomized single blinded case control study. Surg Endosc. 2018 Dec;32(12):4985-4989. doi: 10.1007/s00464-018-6261-6. Epub 2018 Jun 4.

8. Batko I, Kościelniak BK, Al-Mutari I, Kobylarz K. Benefits of ultrasound-guided transversus abdominis plane block for open appendectomy in children. Anaesthesiol Intensive Ther. 2017;49(3):198-203. doi: 10.5603/AIT.a2017.0039. Epub 2017 Aug 2.

9. Fusco P, Cofini V, Petrucci E, Scimia P, Pozone T, Paladini G, Carta G, Necozione S, Borghi B, Marinangeli F. Transversus Abdominis Plane Block in the Management of Acute Postoperative Pain Syndrome after Caesarean Section: A Randomized Controlled Clinical Trial. Pain Physician. 2016 Nov-Dec;19(8):583-591. 